\title{
Análise do Estado Nutricional em Diferentes Fases de Vida Escolar em Escolas de Rede Municipal
}

\author{
Delma Cristina Pereira da Silva, ${ }^{1}$ Mariana Pirani Rocha Machado, ${ }^{2}$ \\ Ingrid Cristina Ferreira da Silva, ${ }^{1}$ Cláudia Pereira Soares Sanchez Lacerda, ${ }^{1}$ \\ Suiani Priscila Roewer, ${ }^{1}$ Eyd Evelyn Alves, ${ }^{1}$ Sara Morgana Forgerini, ${ }^{1}$ \\ Érika Maria Neif ${ }^{1}$
}

\section{RESUMO}

Este estudo teve como objetivo avaliar o estado nutricional em diferentes fases de vida escolar de crianças das escolas da rede municipal do município de Montes Claros - GO. Trata-se de um estudo do tipo observacional descritivo, com corte transversal. Os dados foram obtidos por meio de entrevista, utilizando-se um questionário semiestruturado, com aplicação individualizada. Inicialmente foram coletados dados de idade, e, posteriormente, foi realizada a avaliação antropométrica, caracterizada pela avaliação da estatura e peso da massa corporal, os quais foram verificados por intermédio de fita métrica, estadiômetro, balança digital e calculadora. Os escolares foram divididos em 5 grupos de acordo com a faixa etária estabelecida pelo Programa Nacional de Alimentação Escolar, sendo, destes, 370 (48,62\%) do gênero feminino e 391 (51,38\%) do masculino. Inicialmente os grupos apresentaram uma faixa de eutrofia com $82 \%$ para meninos e 74,13\% para meninas. Com o avanço da idade foi possível observar uma redução de estado nutricional adequado para $61,34 \%$ nos meninos e 55,68\% nas meninas, resultado esse que evidencia a transição nutricional pela qual o país tem passado. A presença do profissional nutricionista, guiados pelas diretrizes, é um fator fundamental para a manutenção do estado nutricional adequado dos escolares.

Palavras-chave: escola; educação nutricional; nutricionista; obesidade.

\section{ANALYSIS OF THE NUTRITIONAL STATE IN DIFFERENT PHASES OF SCHOOL LIFE}

\section{ABSTRACT}

This study had of aimed to evaluate the status nutritional in different stages of school life of children from the municipal school of Montes Claros- GO. This is a study descriptive observational cross-sectional. Data were obtained through interviews using a semi-structured questionnaire with individualized application. Initially, age data were collected, later anthropometric evaluation was performed, characterized by the evaluation of height, weight, body mass index (BMI), which were verified by measuring tape, stadiometer and digital scale and calculator. The students were divided into 5 groups according to the age group established by the Programa Nacional de Alimentação Escolar (PNAE). We find 370 (48.62\%) females and $391(51.38 \%)$ males. Initially, the groups had an eutrophic range of $82 \%$ for boys and $74.13 \%$ for girls. With advancing age, it was possible to observe an adequate reduction in nutritional status to $61.34 \%$ in boys and $55.68 \%$ in girls. This highlights the nutritional transition the country has been through. The presence of the professional nutritionist guided by the PNAE guidelines is a fundamental factor for maintaining the proper nutritional status of the students.

Keywords: anthropometric evaluation; nutritional education; Nutritionist; obesity.

RECEBIDO EM: 26/5/2020

MODIFICAÇÕES SOLICITADAS EM: 25/11/2020

ACEITO EM: $12 / 3 / 2021$

\footnotetext{
${ }^{1}$ Centro Universitário do Vale do Araguaia (Univar). Barra do Garças/MT, Brasil.

2 Autora correspondente. Centro Universitário do Vale do Araguaia (Univar). R. Moreira Cabral, 1000 - Setor Mariano. Barra do Garças/MT, Brasil. CEP 78603-209. http://lattes.cnpq.br/7638132210951257. https://orcid.org/0000-0001-8412-2483. mariana.pirani@hotmail.com
} 


\section{INTRODUÇÃO}

As diretrizes relacionadas à promoção de alimentação saudável nas escolas devem ser instituídas como um elemento a ser contemplado no projeto político pedagógico das unidades escolares (BRASIL, 2006). A efetivação destas ações implica na participação dos profissionais da educação, da comunidade escolar, dos profissionais da saúde e da comunidade local, visando à articulação entre escola e comunidade para uma gestão democrática (AZEVEDO, 2009).

O Ministério da Saúde e o da Educação são corresponsáveis por assegurar a efetivação da Política Nacional de Alimentação e Nutrição (BRASIL, 2012). Consequentemente, de parte da Política Nacional de Promoção de Saúde nas escolas, em consonância com os critérios de execução do Programa Nacional de Alimentação Escolar (PNAE), por sua vez, tem como objetivos atender às necessidades nutricionais do aluno no período escolar, promovendo hábitos alimentares saudáveis (BRASIL, 2006).

O consumo excessivo de calorias promove o desequilíbrio entre o ganho e o gasto de energia, levando a desordens metabólicas, como a obesidade infantil, que, por sua vez, aumenta os riscos de ocorrência de diabetes mellitus tipo 2, intercorrências cardiovasculares e distúrbios psicológicos e comportamentais, como transtorno de déficit de atenção/hiperatividade (TDAH) (ABARCA-GÓMEZ et al., 2017; ZHANG et al., 2019).

O sobrepeso/obesidade infantil é problema de saúde pública atual com índices crescentes no século 21. As estimativas da Organização Mundial de Saúde (OMS) demonstram incidência de 40 milhões de crianças nesta situação em todo mundo, especialmente nos países em desenvolvimento (WHO, 2016; MĂRGINEAN et al., 2019). A estimativa mundial da International Obesity Task Force (IOTF) é de que, atualmente, 155 milhões de crianças estejam com excesso de peso, sendo maior a prevalência de obesidade infantil em países desenvolvidos (IOTF, 2005). A prevalência de sobrepeso e obesidade entre crianças e adolescentes de 5 a 19 anos aumentou drasticamente de apenas 4\% em 1975 para pouco mais de $18 \%$ em 2016, e o crescimento ocorreu de maneira semelhante entre meninos e meninas (WHO, 2020).

Esses índices são preocupantes, o que a torna um problema de saúde global que vem aumentando significativamente em todo o mundo e está relacionada ao estilo de vida atual, caracterizado pelo estresse, o consumo excessivo de alimentos e o sedentarismo, sendo estes os principais fatores relacionados com a gênese dessa doença (MOUCHACCA; ABBOTT; BALL 2013; BREWIS; STURTZSREETHARAN; WUTICH, 2018). Esta é uma condição clínica séria e prevalente, podendo tornar-se o principal problema de saúde do século 21 e a primeira causa de doenças crônicas do mundo (CHOORAMANI; PRASAD; AKBAR, 2018).

No Brasil, pesquisa da Associação Brasileira para Estudos de Obesidade (Abeso) mostra que a obesidade infantil triplicou nos últimos 20 anos (ABESO, 2016). A obesidade infantil está diretamente relacionada a anormalidades na pressão sanguínea, nos níveis de lipídios, lipoproteínas e insulina em adultos, bem como associada aos riscos de doença arterial coronariana e diabetes mellitus (ROCCHINI, 2002).

Editora Unijuí - Revista Contexto \& Saúde - ISSN 2176-7114 - v. 21, n. 43, jul./set. 2021 
Neste sentido, a alimentação escolar está presente nas instituições escolares como disciplina pela Lei no 11.947/2009, incluindo a educação alimentar e nutricional no processo de ensino e aprendizagem que transcorre pelo currículo escolar, abordando o tema alimentação e nutrição e o desenvolvimento de práticas saudáveis de vida, na perspectiva da segurança alimentar e nutricional (BRASIL, 2009).

Considerando a gestão descentralizada da educação, os municípios passam a elaborar planos e projetos governamentais para traduzir em ações as normatizações. Neste processo, são muitos os mediadores que regem as ações até que estas cheguem aos espaços escolares em virtude da sua complexidade e dos diversos atores que ainda não usam plenamente as possibilidades e oportunidades neste processo de educação e saúde concomitantemente (BRASIL, 2009).

Em razão desta problemática, existem riscos quando não se trata sobre a educação alimentar e nutricional no processo de ensino e aprendizagem. Sendo assim, surge a necessidade de novas pesquisas dirigidas a esta população, posto que poucos são os estudos sobre a influência do processo educativo sobre a educação alimentar ao longo da vida escolar de crianças. O presente estudo, portanto, teve como objetivo analisar o estado nutricional em diferentes fases de vida escolar de crianças das escolas da rede municipal do município de Montes Claros-GO.

\section{MATERIAIS E MÉTODOS}

\section{Delineamento e População de Estudo}

Trata-se de um estudo do tipo observacional descritivo, com coorte transversal. Foram incluídos no total 761 participantes, sendo estes estudantes das escolas da rede municipal do município de Montes Claros - GO.

\section{Característica Amostral}

A amostra foi caracterizada com 370 participantes do sexo feminino e 391 do sexo masculino, compreendendo faixa etária entre 2 a 18 anos. Para serem incluídos, os participantes teriam de estar matriculados nas escolas e na idade da tabela do Programa Nacional de Alimentação Escolar (PNAE). Foram excluídos participantes que não atendessem esses requisitos ou que não permitiram a coleta de dados.

\section{Instrumento para a Coleta de Dados}

A coleta de dados foi realizada no período letivo com autorização da direção de cada escola investigada. Os dados foram obtidos por meio de entrevista, quando se utilizou um questionário semiestruturado, e a aplicação ocorreu de forma individual. O período de coleta teve duração de sete dias, sendo intercalados os turnos matutino e vespertino durante o período dos meses de fevereiro, março e abril de 2018. Inicialmente foram coletados dados de idade, massa corporal e estatura; posteriormente foi avaliado o estado nutricional 
usando-se o índice de massa corporal (IMC). Os dados antropométricos foram verificados com fita métrica, estadiômetro, balança digital e calculadora para verificar o IMC.

\section{Análises de Dados}

Os dados coletados foram tabulados, armazenados e analisados em planiIhas no Excel 2007. Os resultados referentes à caracterização da amostra foram apresentados em valores de porcentagem, estratificados por gênero e por grupos. Os grupos foram classificados de acordo com a idade escolar proposta na Resolução CD/FNDE no 26/2013 do PNAE.

\section{Aspectos Éticos}

Todos os responsáveis das escolas foram informados sobre os procedimentos e objetivos do estudo e, após concordarem em participar, foram convidados a assinar o Termo de Consentimento Livre e Esclarecido (TCLE). O Termo de Assentimento Livre e Esclarecido (Tale) foi assinado pelos pais ou responsáveis para participantes menores de 18 anos, assegurando todos os seus direitos.

O estudo foi aprovado pelo Comitê de Ética em Pesquisa (CEP) envolvendo seres humanos da Universidade Federal de Mato Grosso, sob número do Parecer 3.940.014. Reitera-se, ainda, que todas as ações empregadas neste estudo obedecem aos Critérios da Ética na Pesquisa com Seres Humanos, de acordo com a Resolução n. 466/12 do Conselho Nacional de Saúde - Brasília - DF, minimizando riscos e/ou quaisquer desconfortos aos participantes.

\section{Análise Estatística}

Os dados referentes ao estado nutricional foram apresentados em valores de porcentagem referentes a cada domínio verificado e apresentados em gráfico. Já os dados gerais, referentes ao gênero e características antropométricas, estão representados em tabela com valores de média e desvio padrão, considerando diferença estatística quando $p<0,05$.

\section{RESULTADOS}

No total foram incluídos 791 estudantes, matriculados em 7 escolas municipais do município de Montes Claros-GO, sendo, destes, 370 (48,62\%) do gênero feminino e 391 (51,38\%) do masculino. No que se refere às características antropométricas, informações referentes aos valores de mínimo, máximo, média, desvio padrão e $p$-valor foram calculados para variáveis de idade, estatura, peso corporal e IMC por sexo. Desses, não se verificou diferença estatística significante entre os sexos para essas variáveis. As informações descritas, referentes à idade e às características antropométricas dos participantes, são apresentadas na Tabela 1. 
Tabela 1 - Idade e características antropométricas e gerais dos participantes do estudo segundo o sexo. Montes Claros, GO, 2018

\begin{tabular}{|c|c|c|c|c|c|}
\hline \multirow{2}{*}{$\begin{array}{c}\text { Idade e caracterís- } \\
\text { ticas antropomé- } \\
\text { tricas }\end{array}$} & \multicolumn{2}{|c|}{ Feminino } & \multicolumn{2}{|c|}{ Masculino } & \multirow{2}{*}{$p$-valor } \\
\hline & Mín / Máx & $M \pm D P$ & Mín / Máx & $M \pm D P$ & \\
\hline Idades (anos) & $1-15$ & $8,11 \pm 3,1$ & 4- 18 & $8,57 \pm 33,46$ & 0,056 \\
\hline Altura (metros) & $0,71-1,7$ & $1,33 \pm 0,2$ & $1,06-1,83$ & $1,36 \pm 0,22$ & 0,121 \\
\hline Peso (Kg) & $9,4-98$ & $32,59 \pm 14,3$ & $14,4-90,3$ & $34,24 \pm 15,70$ & 0,355 \\
\hline IMC (Kg. $\left.\mathrm{m}^{2}\right)$ & $12,0-46,98$ & $17,58 \pm 3,9$ & $10,75-38,64$ & $17,66 \pm 3,86$ & 0,921 \\
\hline
\end{tabular}

Legenda: Mín = valor mínimo; Máx = valor máximo; $\mathbf{M}=$ média; $\mathbf{D P}=$ desvio padrão e IMC = Índice de massa corporal; $\mathbf{K g}=$ quilograma; $\mathbf{K g} \cdot \mathbf{m}^{2}=$ peso $/$ altura $^{2} ; \mathbf{c m}=$ centímetros. Diferença estatisticamente significativa $(p<0,05)$.

Fonte: Elaborada pelos autores, 2021.

Quanto às informações sobre o estado nutricional de crianças entre 1 e 3 anos, verificou-se no gênero masculino a prevalência de sobrepeso e magreza de $25,92 \%$ e $10 \%$ respectivamente, enquanto no gênero feminino os valores apresentaram-se menores, cerca de 3,70\% e 7,40\% respectivamente. Quanto ao peso corporal adequado, no gênero feminino verificou-se que $62,96 \%$ das avaliadas estão eutróficas, superando os meninos, que correspondem a $60 \%$. Ainda, as meninas demonstraram maior risco de sobrepeso, 25,92\%, em relação aos meninos, 20\%. Estes dados foram apresentados na Figura 1.

Figura 1 - Estado nutricional de crianças entre 1 e 3 anos

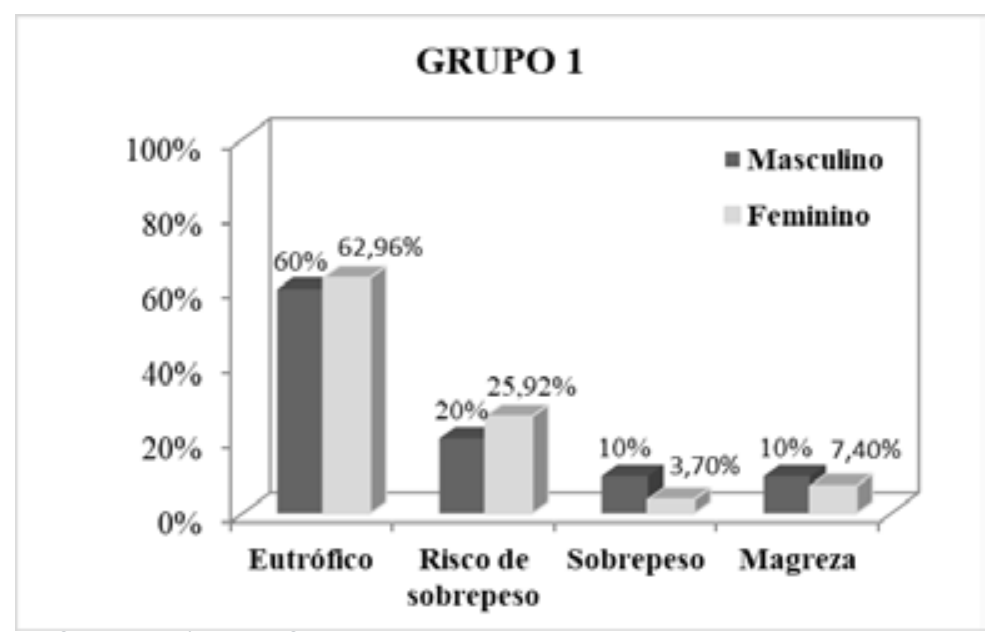

Fonte: Elaborada pelos autores, 2021.

Na Figura 2 observou-se a prevalência de obesidade em crianças entre 4 e 5 anos: $6 \%$ no gênero masculino e 3,44\% no feminino (3,44\%). Em relação ao sobrepeso, notou-se o contrário, uma vez que a prevalência foi de 15,51\% nas meninas e $2 \%$ nos meninos. Ainda, nas meninas verificou-se magreza em $5,17 \%$ destas, enquanto nos meninos não foi observada. Verifica-se ainda que os meninos representam maior risco de sobrepeso em comparação às meninas. Quanto ao peso adequado (eutrofia), percebe-se que os meninos apresentaram melhores índices do que as meninas nessa faixa etária, correspondendo a $82 \%$ e $74,13 \%$, respectivamente. 
Figura 2 - Estado nutricional de crianças entre 4 e 5 anos

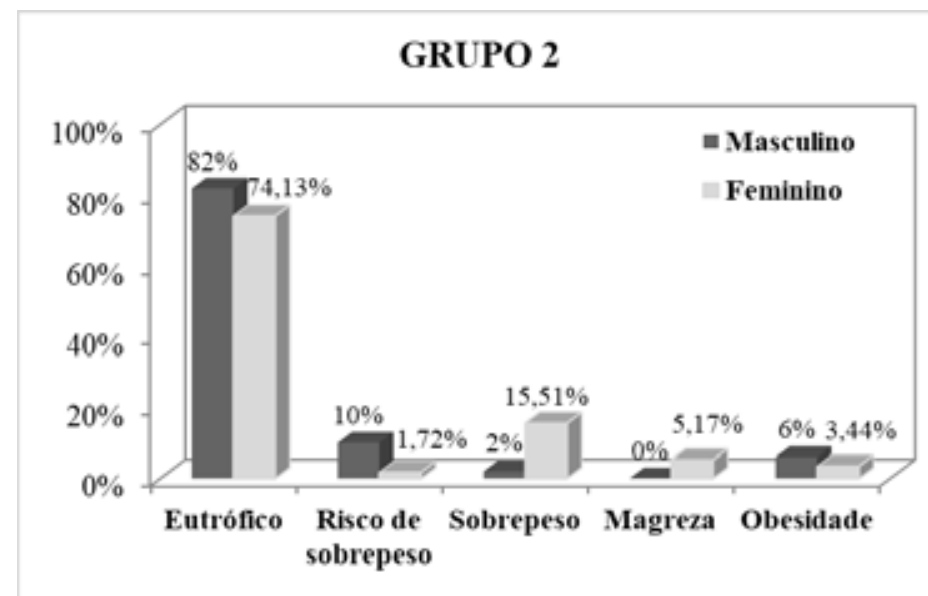

Legenda: $\%$ = porcentagem .

Fonte: Elaborada pelos autores, 2021.

Foi observado no grupo 3 a prevalência de eutrófico em crianças entre 6 e 10 anos: no gênero masculino 59,4\% e no feminino 64,5\%. Em relação ao sobrepeso e obesidade corresponderam, respectivamente, a $18,7 \%$ e $11,8 \%$ nos meninos e $9,3 \%$ e $12,2 \%$ nas meninas. Ainda, nas meninas, verificou-se magreza em $4,1 \%$ destas, enquanto nos meninos foi observado 10,2\% (Figura 3).

Figura 3 - Estado nutricional de crianças entre 6 e 10 anos

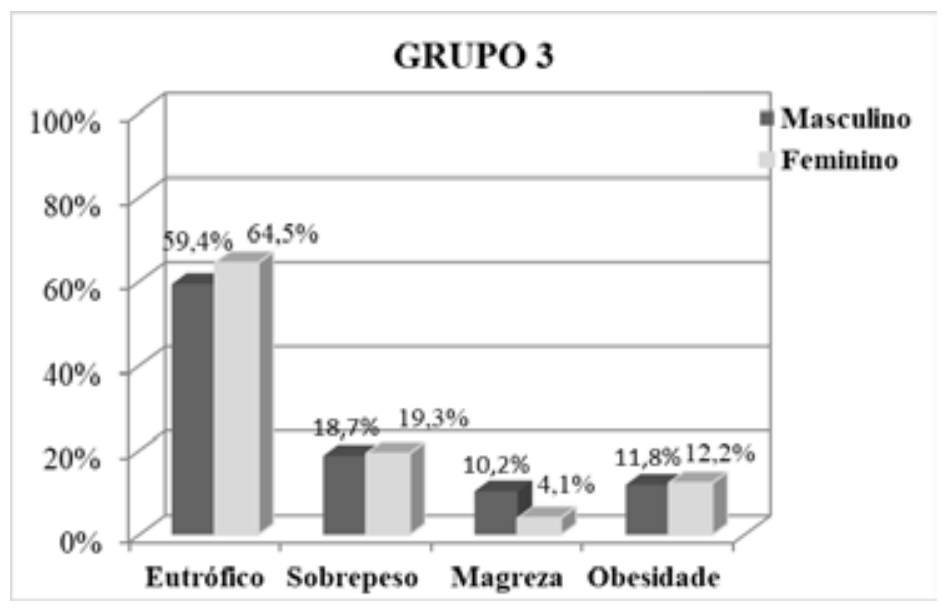

Legenda: \% = porcentagem.

Fonte: Elaborada pelos autores, 2021.

O grupo 4 representa o estado nutricional em adolescentes com faixa etária de 11 a 15 anos. Observa-se maior prevalência de obesidade no gênero masculino, correspondendo a $17 \%$ dos escolares e $11 \%$ do gênero feminino. Já o número de sobrepeso é maior nas meninas, apresentando cerca de $24 \%$, e menor nos meninos, com $17 \%$. Quanto ao peso adequado verificou-se maior porcentagem nos meninos (61\%) do que nas meninas (56\%). Em se tratando dos valores relacionados à magreza, o gênero feminino apresentou um total de $9 \%$, enquanto os meninos $4 \%$. 
Figura 4 - Estado nutricional de crianças entre 11 e 15 anos

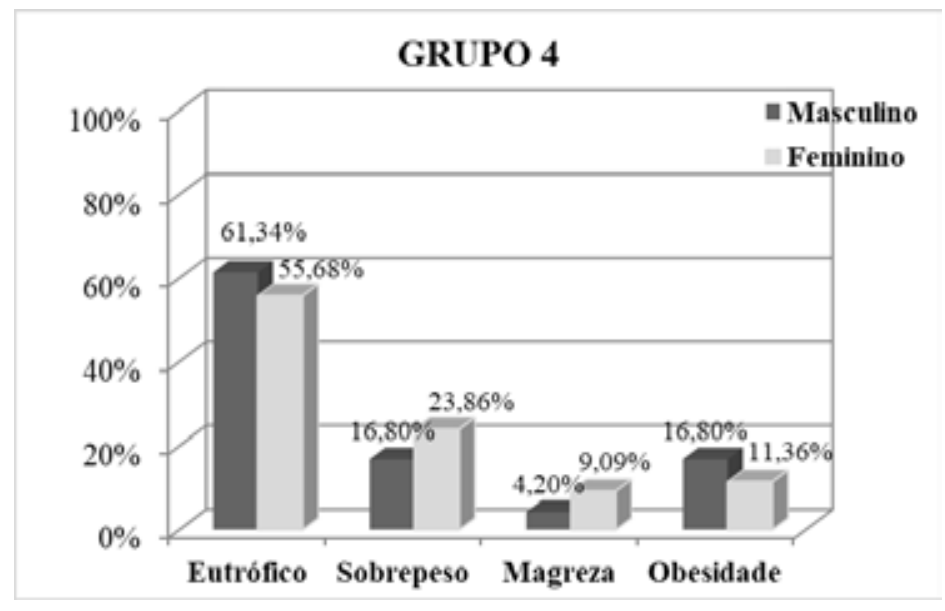

Legenda: \% = porcentagem .

Fonte: Elaborada pelos autores, 2021.

No grupo 5 foi possível avaliar a prevalência de obesidade em escolares do gênero masculino na faixa etária entre 16 e 18 anos. Nota-se que os valores quanto à eutrofia foram de $66,66 \%$ dos alunos avaliados, enquanto apenas $33,33 \%$ estão dentro da faixa de obesidade.

Figura 5 - Estado nutricional de crianças entre 16 e 18 anos

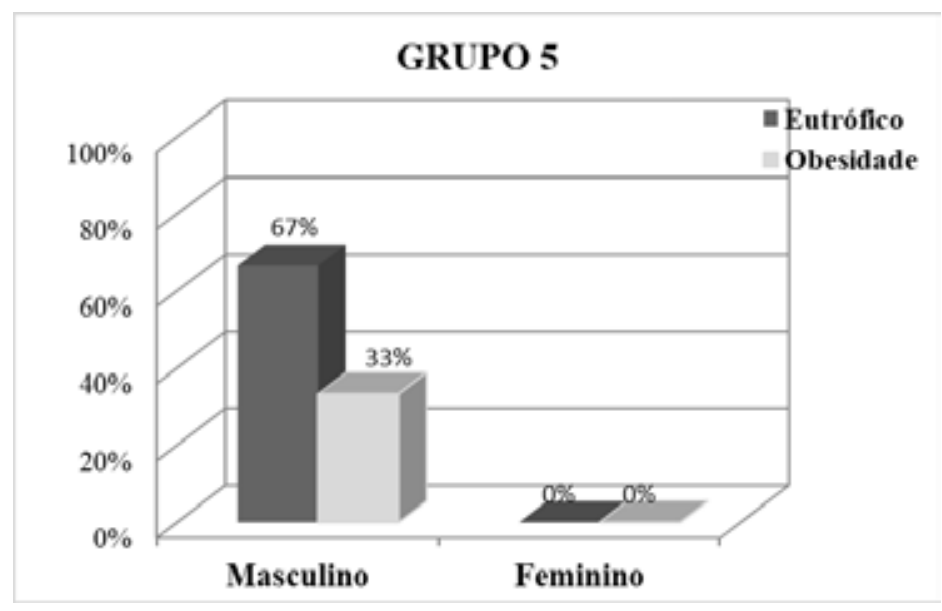

Legenda: \% = porcentagem .

Fonte: Elaborada pelos autores, 2021.

\section{DISCUSSÃO}

A avaliação do estado nutricional da criança ou adolescente envolve a aferição de peso (Kg), idade, altura (m) e Índice de Massa Corporal (IMC). Acompanhar o crescimento infantil é desenvolver a vigilância nutricional adequada, prevenindo problemas de má nutrição, obesidade e outras doenças associadas à alimentação. $\mathrm{O}$ estado nutricional adequado do escolar garante, sobretudo, o desenvolvimento de suas funções cognitivas e de uma aprendizagem com maior precisão quando comparada à criança má nutrida (ELIAS; GARRIDO JR., 2011), 
posto que a nutrição adequada nos primeiros anos de vida é fundamental para o crescimento e o desenvolvimento saudáveis (DE CARVALHO et al., 2015).

O estado nutricional infantil está diretamente ligado a questões economicamente sociais, como o acesso a alimentos saudáveis e informações. Inadequações no consumo de nutrientes podem comprometer o estado nutricional e levar ao desenvolvimento de carências ou excessos nutricionais (CAVALCANTE et al., 2006; DE CARVALHO et al., 2015), acarretando distúrbios metabólicos, como a obesidade. A obesidade tem características multifatoriais em que estão envolvidos fatores biológicos e socioeconômicos, no entanto o principal ponto é o balanço energético positivo oriundo do aumento da ingestão de alimentos e bebidas calóricas excedendo o gasto de energia; assim, a manutenção deste estado por tempo prolongado resultará em sobrepeso e obesidade (PRINZ, 2019).

Os índices de obesidade na infância e na adolescência têm aumentado de forma significativa (ABARCA-GÓMEZ et al., 2017) em todo o globo. De acordo com a Organização Mundial de Saúde, uma em cada dez crianças em todo o mundo é obesa, o que representa cerca de 155 milhões, e ainda informa que a prevalência global de obesidade pediátrica aumentou para > 5\% entre 1975 e 2016 (YANOVSKI, 2018). Assim, a ingestão alimentar não saudável, disseminada com auxílio da mídia, favorece a formação de um espaço obesogênico, propiciando a fome oculta, e não suprindo as necessidades nutricionais necessárias (PINHEIRO; CARVALHO, 2010).

Sabe-se que a prevalência da obesidade infantil é, hoje, um problema mundial de saúde pública, por isso é de extrema importância o acompanhamento nutricional das crianças nas escolas feita por um nutricionista, que é o profissional qualificado para avaliar e tomar as medidas cabíveis para a recuperação do estado nutricional (WHO, 2016). Existem indícios e inúmeros estudos que apontam a relação entre o merchandising e a preferência alimentar das crianças. Tal fator influencia diretamente na dieta infantil, promovendo intenso consumo de alimentos pobres em nutrientes e ricos em calorias, como comidas rápidas, cereais matinais e bebidas calóricas (CARTER et al., 2011).

No estudo de Santos et al. (2019), por exemplo, os autores observaram aumento na prevalência de sobrepeso e obesidade entre estudantes do Estado de Santa Catarina entre os anos de 2001 e 2011, e entre os fatores sociodemográficos investigados, meninos e meninas que vivem em áreas urbanas e com alta renda mensal bruta apresentaram, na pesquisa de 2011, maiores chances de estar acima do peso e obesos em comparação com a pesquisa de 2001.

Ainda, de acordo com um estudo realizado por Lourenço et al. (2019) em pré-escolas de Macaé, o ambiente escolar influencia no estado nutricional das crianças. A escola com mais lanchonetes e propagandas de alimentos no território teve maior proporção de obesidade, principalmente entre crianças menores de 5 anos, e, ainda, fatores positivos de ambiência dentro da escola, como ausência de cantina comercial, não conseguiram evitar o excesso nutricional.

No âmbito da população brasileira houve uma mudança nutricional ocorrida nas últimas décadas, com redução da subnutrição e elevação de sobrepeso e obesidade infantil. Tais características, além de poderem acarretar comor-

Editora Unijuí - Revista Contexto \& Saúde - ISSN 2176-7114 - v. 21, n. 43, jul./set. 2021 
bidades à saúde, aumentam as chances de crianças obesas tornarem-se na vida adulta indivíduos obesos (FLORES et al., 2013). Esta transição nutricional no Brasil foi fortemente demarcada pela saída de um quadro de fome e insegurança alimentar para um panorama de excesso de peso e doenças crônicas não transmissíveis (VAZ, 2018).

A sanção da Lei Orgânica de Segurança Alimentar e Nutricional (Losan), em 2006, foi um fator essencial para a garantia do Direito Humano à Alimentação Adequada (DHAA) como direito humano fundamental. A partir desse direito social foram criados e executados diversos programas alimentares visando à garantia e exercício desse direito; um dos mais importantes é o PNAE (GARCIA; CARNIATTO; GRANDI, 2018).

O PNAE é popularmente conhecido como merenda escolar; é gerenciado pelo Fundo Nacional de Desenvolvimento da Educação (FNDE) e visa à oferta de refeições adequadas de acordo com a faixa etária e as necessidades nutricionais dos alunos. É considerado um dos maiores programas na área de alimentação escolar no mundo e é o único com atendimento universalizado. A partir de 2006 houve importante conquista no contexto do PNAE, quando foi exigida a presença do nutricionista como responsável técnico. Não obstante, em 2009, com o Programa, ocorreu um grande avanço com a inclusão dos alunos participantes do Programa Mais Educação e de Jovens e Adultos, além da garantia da aquisição de produtos advindos da agricultura família (BRASIL, 2019).

As atribuições do nutricionista do PNAE vão muito além do cálculo de cardápios de acordo com as necessidades, fator cultural e oferta de alimentos advindos da agricultura familiar. O nutricionista promove atividades de educação nutricional para influenciar bons hábitos nutricionais e um estilo de vida mais saudável, estando diretamente ligado ao estado nutricional das crianças (BRASIL, 2019).

No presente estudo foi notória a prevalência da eutrofia entre os escolares em todas as faixas etárias pesquisadas. Em seguida, foi possível observar um aumento crescente do sobrepeso e obesidade na medida em que se avançava a idade dos escolares. Este fato pode estar relacionado à fase conhecida como estirão puberal, em que ocorre o acúmulo de gordura prévio, com um ganho aproximado de $50 \%$ do que seria seu peso quando adulto. Este mesmo quadro foi observado de forma similar no estudo realizado por Izidoro, Santos e Oliveira (2014).

Um fator que pode justificar a maior prevalência de eutrofia entre os escolares é a importante atuação do profissional nutricionista guiado pelas recomendações do PNAE no município de Montes Claros-GO. Por meio do Programa a nutricionista desenvolve atividade de educação nutricional voltada para a família de forma integral, com pais e escolares envolvidos no processo de educação. Foram desenvolvidas atividades interativas, como gincanas, palestra e reunião com os pais dos escolares.

Apesar de a presença do nutricionista no ambiente escolar ser um ponto favorável, a prevalência da eutrofia também se justifica por outros motivos. A eutrofia se mantém, pois o adequado aporte nutricional potencializa o desen-

Editora Unijuí - Revista Contexto \& Saúde - ISSN 2176-7114 - v. 21, n. 43, jul./set. 2021 
volvimento adequado da criança em suas atividades diárias, visto que a alimentação que não supre ou que excede as necessidades individuais dificulta o bom desempenho das funções biológicas (IZIDORO; SANTOS; OLIVEIRA, 2014.) A mídia, o ambiente social e a conduta familiar nos momentos das refeições mostraram-se fundamentais na determinação do comportamento alimentar de escolares e no desenvolvimento de seus transtornos nutricionais (GONÇALVES et al., 2013).

O nutricionista é o profissional licenciado que assume toda a sistematização das ações do planejamento, coordenação, direção, supervisão e avaliação na área da alimentação e nutrição dentro do âmbito educacional escolar, por meio das esferas municipal, estadual e distrital, sendo o responsável técnico do Programa Nacional de Alimentação Escolar (PNAE), promovendo a articulação estruturante com a área da educação. Esse profissional contribui, ainda, na construção de um Plano Político Pedagógico (PPP), propondo o estabelecimento de novos paradigmas em gestão e em práticas pedagógicas que avaliza à instituição escolar transgredir a denominada "educação tradicional", cujo exercício de base positivista mostrar-se aquém de responder às indigências e aspirações dos atores principais: os alunos. Dessa maneira, compõe-se na escola um ambiente propício a promoção e prevenção da saúde e da educação.

Em se tratando das atividades obrigatórias do profissional nutricionista, é imprescindível a observação de atribuições complementares e de outras que poderão ser desenvolvidas de acordo com a necessidade, complexidade dos serviços e disponibilidade da estrutura operacional. Nesse aspecto, ressalta-se que os resultados aqui relatados foram apropriados conforme o planejamento e o desenvolvimento de ações, uma vez que todos os profissionais receberam treinamento, como a formação continuada, assessoria aos municípios e capacitações de gestores, nutricionistas, conselheiros de alimentação escolar, agricultores familiares, entre outros atores do PNAE.

O presente estudo reforça a importância de ações conjuntas entre os nutricionistas e os demais membros da comunidade escolar junto ao poder público local, com vistas a contribuir efetivamente com a garantia do Direito Humano à Alimentação Adequada e Saudável e a Segurança Alimentar e Nutricional.

\section{CONCLUSÃO}

Neste estudo foram analisados 791 estudantes matriculados em escolas municipais de Montes Claros-GO, sendo, destes, $48,62 \%$ do gênero feminino e $51,38 \%$ do masculino. Não houve diferença estatística significativa entre os gêneros para as variáveis analisadas: idade, estatura, peso corporal e IMC. Quanto ao estado nutricional, nas crianças com faixa etária entre 1 e 3 anos verificou-se a prevalência de sobrepeso no sexo masculino, enquanto entre 4 e 5 anos observou-se o contrário. Na faixa etária de 6 a 18 anos novamente a prevalência foi no sexo masculino.

Conclui-se que, perante os resultados encontrados, houve cumprimento dos princípios básicos do Programa Nacional de Alimentação Escolar (PNAE), posto que foi preservada a qualidade em relação à quantidade da alimentação

Editora Unijuí - Revista Contexto \& Saúde - ISSN 2176-7114 - v. 21, n. 43, jul./set. 2021 
que é ofertada aos escolares, a efetividade na elaboração das fichas técnicas de preparação, respeitando a análise da composição nutricional, e a aceitação e efetiva ingestão por parte dos alunos, o que pode indicar situação do cumprimento das responsabilidades técnicas do nutricionista.

Além disso, tais achados indicam a efetividade do cumprimento das normativas do PNAE por parte desse município e da adoção de medidas corretivas, a fim de resolver as possíveis inadequações. Nesse aspecto, destaca-se a importância da figura do nutricionista e a efetiva valorização do profissional enquanto gestor e responsável por um dos maiores programas de promoção da Segurança Alimentar e Nutricional do país.

\section{CONFLITOS DE INTERESSE}

Nenhum declarado. Os autores informam que a pesquisa foi conduzida sem características que pudessem ser interpretadas como um potencial conflito de interesses.

\section{AGRADECIMENTOS}

Os autores gostariam de agradecer aos voluntários da pesquisa bem como aos diretores das escolas e à Prefeitura de Montes Claros-GO.

\section{REFERÊNCIAS}

ABARCA-GÓMEZ, L. et al. Worldwide trends in body-mass index, underweight, overweight, and obesity from 1975 to 2016: a pooled analysis of 2416 population-based measurement studies in 128.9 million children, adolescents, and adults. The Lancet, v. 390, n. 10.113, p. 2.627-2.642, 2017.

ABESO. Associação Brasileira para Estudos da Obesidade. Diretrizes Brasileiras para a Obesidade, Abeso, 4, 2016.

AZEVEDO, J. M. Programas federais para a gestão da educação básica: continuidade e mudanças. Rev. Bras. Pol. Adm. Escol., (25), p. 211-231, 2009.

BRASIL. Fundo Nacional de Desenvolvimento da Educação. Resolução FNDE/ CD no 32, de 10 de agosto de 2006. Estabelece as normas para a execução do Programa Nacional de Alimentação Escolar. Brasília: Diário Oficial da União, 10 ago. 2006.

BRASIL. Presidência da República. Lei no 11.947, de 16 de junho de 2009. Dispõe sobre o atendimento da alimentação escolar e do Programa Dinheiro Direto na Escola aos alunos da educação básica. Brasília: Diário Oficial da União, 16 jun. 2009.

BRASIL. Ministério da Saúde. PNAN: Política Nacional de Alimentação e Nutrição. Brasília: MS, 2012.

BRASIL. Ministério da Saúde. Programa Nacional de Alimentação Escolar (PNAE). Caderno de Legis/ação. Brasília: MS, 2019.

BREWIS, A.; STURTZSREETHARAN, C.; WUTICH, A. Obesity stigma as a globalizing health challenge. Globalization and Health, (14), p. 1-20, 2018.

CARTER, M. A. et al. Availability and marketing of food and beverages to children through sports settings: a systematic review. Public Health Nutr, 15(8), p. 1.373-1.379, 2011.

CAVALCANTE, A. A. M. et al. Consumo alimentar e estado nutricional de crianças atendidas em serviços públicos de saúde do município de Viçosa, Minas Gerais. Revista de Nutrição, v. 19, n. 3, p. 321-330, 2006. 
CHOORAMANI, G.; PRASAD, B. V.; AKBAR, S. Obesity Physical and Mental Health Consequences in Ageing. Handbook of Research on Geriatric Health, Treatment, and Care, p. 288-302, 2018. Disponível em: https://www.igi-global.com/chapter/obesity-physical-and-mental-health-consequences-in-ageing/201387.

DE CARVALHO, C. A. et al. Consumo alimentar e adequação nutricional em crianças brasileiras: revisão sistemática. Rev. Paulista de Pediatria, v. 33, n. 2, p. 211-221, 2015.

ELIAS, A. A.; GARRIDO JR., B. Derivações gástricas em y- de- roux com anel de silicone para o tratamento da obesidade. Arq. Bras. Cir. Dig., 24(4), p. 290-295, 2011.

FLORES, L. S. et al. Tendência do baixo peso, sobrepeso e obesidade de crianças e adolescentes brasileiros. J. de Pediatria, 89(5), p. 456-461, 2013.

GONÇALVES, J. A. et al. Transtornos alimentares na infância e na adolescência. Rev. Paulista de Pediatria, v. 31, n. 1, p. 96-103, 2013.

IOTF. International Obesity Task Force. The Report of the National Taskforce on Obesity 2005. Obesity The Policy Challenges, IOTF, 2005.

IZIDORO, G. S. L. et al. A influência do estado nutricional no desempenho escolar. Rev. Cefac, (16), p. 1.541-1.547, 2014.

LOURENÇO, A. E. P. et al. Influência da ambiência escolar no estado nutricional de pré-escolares de Macaé, Rio de Janeiro, Brasil. Cien. e Saúde Colet., v. 24, n. 7, p. 2.399-2.409, 2019. Disponível em: https://www.scielo.br/j/csc/a/nKP7hcYhn3vRXh6JzBkgbCR/?lan$\mathrm{g}=\mathrm{pt}$.

MĂRGINEAN, C. O. et al. The relationship between MMP9 and ADRA2A gene polymorphisms and mothers-newborns' nutritional status: an exploratory path model (STROBE compliant article). Pediatric. Resec., (85), p. 822-829, 2019.

MOUCHACCA, J.; ABBOTT, G. R.; BALL, K. Associations between psychological stress, eating, physical activity, sedentary behaviours and body weight among women: A longitudinal study. BMC Public Health, (13), p. 828, 2013.

PINHEIRO, A. R. O.; CARVALHO, M. F. C. C. Transformando o problema da fome em questão alimentar e nutricional: uma crônica desigualdade social. Ciênc. Saúde Coletiva, v. 15, n. 1, p. 121-130, 2010.

PRINZ, P. The role of dietary sugars in health: molecular composition or just calories? Europ. J. of Clin. Nutr., (73), p. 1.216-1.223, 2019.

ROCCHINI, A. P. Childhood obesity and a diabetes epidemic. New. Engl. J. Med., (346), p. 854-855, 2002.

SANTOS, P. C. et al. Change in overweight and obesity over a decade according to sociodemographic factors in Brazilian adolescents. Cien. e Saúde Colet., v. 24, n. 9, p. 3.335-3.344, 2019. Disponível em: https://www.scielo.br/j/csc/a/KkS8rGYBcbzKRXQTnYnYMtS/?lang=en.

VAZ, Beatriz Abu Ali. Políticas de combate à fome e Programas em nutrição: um olhar da década de 30 até 2018. Comun. em Ciências da Saúde, v. 29, n. 2, p. 126-138, 2018.

WHO. World Health Organization. Report of the Commission on Ending Childhood Obesity. Implementation plan: executive summary. Geneva: WHO, 2016.

WHO. World Health Organization. Obesity and overweight. 2020. Acesso em: maio 2020. Disponível em: http://www.who.int/news-room/fact-sheets/detail/obesity-and-over weight.

YANOVSKI, J. A. Obesity: Trends in underweight and obesity: scale of the problem. Nature Rev. Endoc., 14, p. 5, 2018.

ZHANG, A. et al. Nocturnal enuresis in obese children: a nation-wide epidemiological study from China. Scien. Reports, (9), p. 8.414, 2019. 